\title{
Erratum to: Histological Characterization of Human Breast Implant Capsules
}

\author{
Janine M. Bui ${ }^{1} \cdot$ TracyAnn Perry $^{1} \cdot$ Cindy D. Ren $^{1} \cdot$ Barbara Nofrey $^{1} \cdot$ \\ Steven Teitelbaum ${ }^{2} \cdot$ Dennis E. Van Epps ${ }^{1}$
}

Published online: 16 April 2015

(C) Springer Science+Business Media New York and International Society of Aesthetic Plastic Surgery 2015

\section{Erratum to: Aesth Plast Surg DOI 10.1007/s00266-014-0439-7}

In Table 2, the "d" at the end of "Synovial metaplasiad" and the "e" at the end of "Loosely packed collagene" have been changed from regular text to superscript. The correct version of the table is reprinted below. Springer regrets the error.

The online version of the original article can be found under doi: 10 . 1007/s00266-014-0439-7.

Dennis E. Van Epps

devanepps@cox.net

1 Allergan, Inc., 2525 Dupont, Irvine, CA 92612, USA

2 Division of Plastic and Reconstructive Surgery, David Geffen School of Medicine at UCLA, Los Angeles, CA, USA 
Table 2 Summary of uncontracted versus contracted analysis of capsules

\begin{tabular}{|c|c|c|c|c|c|c|c|}
\hline \multirow[t]{2}{*}{ Capsule characteristic } & \multicolumn{2}{|l|}{ Uncontracted } & \multicolumn{2}{|l|}{ Contracted } & \multirow[t]{2}{*}{ Uncontracted } & \multirow[t]{2}{*}{ Contracted } & \multirow[t]{2}{*}{$p$ value $^{a}$} \\
\hline & Baker I & Baker II & Baker III & Baker IV & & & \\
\hline Thickness $(\mu m)$, mean $\pm S D$ & $91.5 \pm 30.3$ & $408.6 \pm 28.9$ & $393.4 \pm 24.5$ & $355.4 \pm 17.9$ & $285.3 \pm 270.3$ & $389.8 \pm 169.4$ & 0.0111 \\
\hline $\begin{array}{l}\text { Collagen fiber alignment (angle } \\
S D \text { ), mean } \pm S D\end{array}$ & $30.3 \pm 5.6$ & $28.9 \pm 9.5$ & $24.5 \pm 8.3$ & $17.9 \pm 3.1$ & $29.4 \pm 8.1$ & $23.8 \pm 8.2$ & 0.0068 \\
\hline$\alpha-S M A, \%$ of positive samples & 17 & 9 & 39 & 33 & 12 & 39 & 0.049 \\
\hline \multicolumn{8}{|c|}{ Histopathology of capsule, \% of samples } \\
\hline \multicolumn{8}{|l|}{ Dense collagen } \\
\hline Acellular $^{\mathrm{b}}$ & 43 & 55 & 75 & 67 & 50 & 81 & 0.001 \\
\hline Cellular & 14 & 18 & 14 & 33 & 17 & 10 & NS \\
\hline Synovial metaplasia $^{\mathrm{d}}$ & 29 & 9 & 7 & 0 & 17 & 6 & NS \\
\hline Loosely packed collagen $^{\mathrm{e}}$ & 43 & 27 & 11 & 0 & 33 & 10 & NS \\
\hline
\end{tabular}

$N S$ not significant

${ }^{a} p$ value for comparison of uncontracted (Baker I and II) versus contracted (Baker III and IV)

b Example shown in Fig. 4a

c Example shown in Fig. 4b

${ }^{d}$ Example shown in Fig. 4c, d

e Example shown in Fig. 4e, $\mathrm{f}$ 